Original research

\title{
The effect of physical education lesson intensity and cognitive demand on subsequent learning behaviour
}

\author{
Christina H.H.M. Heemskerk ${ }^{\text {a, }}$ *, David Lubans ${ }^{\text {b }}$, Steve Strand ${ }^{\text {a }}$, Lars-Erik Malmberg ${ }^{\text {a }}$ \\ axford University, Department of Education, United Kingdom \\ b Priority Research Centre for Physical Activity and Nutrition, University of Newcastle, Australia
}

\section{A R T I C L E I N F O}

\section{Article history:}

Received 1 July 2019

Received in revised form 14 November 2019

Accepted 12 December 2019

Available online $\mathrm{xxx}$

Keywords:

On-task

Acute exercise

Attention

Physical activity

Cognitive engagement

Primary school

\begin{abstract}
A B S T R A C T
Objectives

To investigate the effect of (i) physical education (PE) lesson intensity and (ii) skill complexity, and (iii) their interaction on students' on-task behaviour in the classroom.

Design

Within-subject repeated-measures Methods

Participants were children $(\mathrm{N}=101$, age 7-11) recruited from four elementary schools in Oxfordshire, United Kingdom. The experiment consisted of manipulating the aerobic intensity (low/medium/high) and skill complexity (low/high) of PE lessons. Children participated in all six conditions of the experiment: low intensity-low complexity (flexibility), medium intensity-low complexity (health related exercise), high intensity-low complexity (sprinting games), low intensity-high complexity (bi-lateral ball skills), medium intensity-high complexity (ball games), high intensity-high complexity (aerobics). Children's behaviour in the classroom was observed every $30 \mathrm{~s}$ for $25 \mathrm{~min}$ before and after each PE lesson and rated as on-task or off-task. Results

A main effect of intensity on children's on-task behaviour was found $\left(\mathrm{F}_{(2,51634)}=11.07, \mathrm{p}<0.001\right)$, with greater on-task behaviour following high intensity PE lessons $\left(\mathrm{t}_{\text {high }}=2.85, \mathrm{p}<0.01-\mathrm{t}_{\text {medium }}=0.52, \mathrm{p}=0.60\right.$, $\mathrm{d}=0.2)$. No main effect of complexity on on-task behaviour was observed $\left(\mathrm{F}_{(1,51636)}=1.89, \mathrm{p}=0.17\right)$. The interaction of intensity and complexity was significant $\left(\mathrm{F}_{(2,51628)}=69.19, \mathrm{p}<0.001\right)$. Conclusions

These findings suggest that participation in PE lessons can improve children's on-task behaviour in the classroom. PE lessons involving high complexity and high intensity, or low complexity and medium intensity appear to have the greatest benefits for students' behaviour in the classroom.
\end{abstract}

(C) 2019

\section{Practical implication}

- Careful planning of PE lesson content can maximise the benefits to pupils' learning.

- Primary school teachers can use PE lesson content to help improve pupils' task-related behaviour.

- No evidence was found of negative effects of PE lesson participation on subsequent classroom behaviour.

\section{Introduction}

Participation in physical activity (PA) can enhance children's cognitive function and academic performance. ${ }^{1,2}$ A range of neurobiological, behavioural and psychosocial mechanisms may explain

\footnotetext{
* Corresponding author.

Email address: Christina.heemskerk@education.ox.ac.uk (C.H.H.M. Heemskerk)
}

these effects. ${ }^{3}$ This study investigates the effects of PA on subsequent classroom behaviour; behavioural engagement is an antecedent of academic achievement ${ }^{4}$ and therefore of interest to teachers. Neurobiological mechanisms thought to explain the link between PA and cognitive performance are based on the overlap in the brain areas involved in motor tasks and cognitive processing for learning and attention. ${ }^{5}$ The effect of acute PA on the brain varies by the intensity and complexity of the exercise. Complex skills require greater involvement of the prefrontal structures, thus providing greater overlap with cognitive processing. ${ }^{6}$ Intensity influences the level of arousal in the brain tissue; low and medium intensity are considered most effective for learning when this is tested immediately after a bout of PA, yet after a delay very low intensity PA has negligible effects. ${ }^{7}$

Results of recent studies investigating the effects of cognitively engaging PA on measures of attention have been inconclusive, ${ }^{8}$ and despite the increase in research being conducted in this area, the dose-response relationship between exercise and on-task behaviour in primary school children remains unclear. The studies with this 
age group tend to focus on comparing exercise to a resting condition, or cognitively engaging exercise to simple exercise at a set intensity. In this study, the effects of low, medium and high intensity exercise with and without cognitive demand from skill complexity on on-task behaviour in the primary school classroom were compared. To the authors' knowledge, no work has been published comparing the combination of exercise intensity and complexity by systematically manipulating physical education (PE) lesson content across both complexity and intensity. To address the lack of ecological validity in the current literature, this study took place in a school setting, with observations conducted during normal classroom lessons, and with the exercise conditions lasting for the duration of a standard PE lesson $($ mean $=39.5 \mathrm{~min})$.

\section{Methods}

Ethical approval was granted by the Departmental Research Ethics Committee at the University of Oxford Department of Education, Oxford, United Kingdom. Head teachers and parents provided written informed consent, and participants provided written informed assent. The study is registered with the Research Registry (research registry 4714).

Thirty-eight elementary schools in Oxfordshire were invited via email to participate in the study, four schools responded and a total of eight classes were recruited. Children in years 3-6 (ages 7-11) were eligible to participate if they were physically capable of taking part in PE lessons, had no diagnosis of epilepsy, ADHD or ASD, and did not require a steroid inhaler for asthma or insulin treatment for diabetes. Due to the small sample size anticipated for this study, pupils with these conditions or medications were excluded to avoid the behaviour findings being influenced by their medical conditions or medication timings, rather than by the intervention effects.

Exercise condition order was randomly allocated at the class level. All children in the class took part in the classroom and PE lessons, but only participants with parental consent wore accelerometers and were observed. Teachers were blind to the study hypotheses and were not informed how PE content related to the research questions. Participants were blind to the specific nature of the observations. To prevent anticipation effects in the lesson before PE, participants were not told what they would be doing in the PE lesson until they had changed into their kit at the start of PE.

Participants were fitted with a GENEActiv ${ }^{9}$ accelerometer at the end of school on the day before the intervention. They were instructed to wear the device on their non-dominant wrist until the end of the next school day. All observations and PE lessons took place in the afternoon. Pre-PE observations started as soon as the children returned to the classroom after lunch and lasted for $25 \mathrm{~min}$. Immediately after observations finished, the class prepared for PE. PE lessons lasted between 31 and $48 \mathrm{~min}(\mathrm{M}=39.5, \mathrm{SD}=4.5)$, depending on the timings of the day in each school. After PE, children returned straight to the classroom without changing, and another 25-min observation took place.

Classroom lesson were taught by the usual classroom teacher, and content varied across participating classes. Teachers were asked to teach the same topic for each of the six weeks to prevent effects of participants' general subject liking and interest on their behaviour. PE lessons were taught by the lead researcher in all classes. They involved low or high skill complexity, and were at low, medium, or high intensity (Fig. S1). Intensity was varied by the level of continuity of exercises, posture, and explosiveness of the movements included. Complexity was achieved by using coordinative exercises ${ }^{10}$ involving bilateral movements, the use of non-dominant hand or foot, sequencing of movements, and frequent and rapid changes to the exercises and movements.

Low intensity, low complexity PE lesson: after a walking-pace warm-up, participants were led through static stretching exercises and completed a sit-and-reach test. They then did additional static stretching and re-did the sit-and-reach test, trying to beat their previous score. Whilst waiting their turn for the sit-and-reach test, they were instructed to remain seated in a designated area and chatted quietly with their peers.

Medium intensity, low complexity lesson: after a simple warm-up involving running to markers, sprinting on the spot, and static stretching, participants completed fitness tests. A timed $20 \mathrm{~m}$ sprint, 1-min press-up test, 1-min sit-up test, and standing long jump were assessed. Each child did the $20 \mathrm{~m}$ sprint three times, and the whole class was given two minutes to independently practise the standing long jump. Sit-ups and press-ups were completed with a partner.

High intensity, low complexity lesson: after playing a high-paced running game and static stretching for their warm-up, participants had a quick practise of simple running technique. They then completed the multi-stage fitness test, capped at level 10. After they failed to keep up with the test pace children were encouraged to keep running on the side lines. Then, continuous $10 \mathrm{~m}$ sprints were completed in a 4-lane course, as well as straight-line relays using high-fives instead of batons.

Low intensity, high complexity lesson: after a warm-up of stationary and walking-pace single-handed throwing a catching of tennis balls with their non-dominant hand and static stretching, participants attempted to juggle two balls for one minute and counted how many times they successfully threw and caught the ball. They then worked with a partner to do exercises involving timing, reaction time, passing and receiving with hands and feet, using predominantly their non-dominant hand or foot (depending on skill level). After five minutes of specific juggling practice they re-did their one minute of juggling, attempting to beat their previous score.

Medium intensity, high complexity lesson: after a running game involving reacting to a partner's movements and static stretching for the warm-up, participants practised their passing and catching skills with their non-dominant hand. They started off stationary, progressing to passing on the move, then passing on the move with a defender (dynamic 'piggy in the middle'). Finally, they played a tournament of 1 -min passing games in teams of three or four, with different rules in each game.

High intensity, high complexity lesson: after a warm-up of free-style moving to music, during which the children were encouraged to be as energetic as possible, and static stretching, the class took part in an aerobics session led by the lead researcher. Movements got increasingly more complex, with arms and legs moving simultaneously in different directions, leg movement staying the same with arm movements changing, and the introduction of turns and jumps. Sequences were repeated for the duration of a full song to ensure high intensity.

Each lesson was concluded with a plenary in which pupils quietly reflected on what they had really enjoyed in the lesson and what they wanted to get better at, and finally they told another child something they had done well in this lesson. They then lined up and returned straight to their classroom for their next lesson and observation.

The observation protocol was adapted from the BOSS. ${ }^{11}$ In this study, on-task behaviour was operationalised as 'goal-directed, task-appropriate behaviour'. The 'goal-directed' element of the operationalisation accounts for the absence or irrelevant or distracted behaviour such as yawning, day-dreaming, task-unrelated movement, or communication that interfered with the completion of the teacher- 
specified task. Two raters conducted the observations, observing six participants each. Across the six weeks, the two raters observed the same participants each time as far as absences allowed. Inter-rater reliability checks were performed at the start of each block of data collection $\left(\mathrm{K}=0.8\right.$, strong agreement $\left.{ }^{12}\right)$. Each participant was observed every $30 \mathrm{~s}$, and behaviour was recorded as 'on-task', 'passive off-task', or 'active off-task'. Task type was also recorded (individual, paired, group work, teacher-led). Off-task behaviour was coded 'passive' or 'active', to assess if the type of off-task behaviour might be affected by exercise intensity or complexity. No further distinction was made between active or passive on-task behaviour, and motor or verbal active off-task behavior. ${ }^{11}$

On-task behaviour was analysed as a binary outcome $(0=$ off-task, $1=$ on-task), using generalised linear mixed models in SPSS $24 .{ }^{13} \mathrm{Ob}-$ servations were nested in days, and days in participants, with pre/ post-PE, PE intensity, and PE skill complexity as predictors of on-task behaviour. Lessons with a minimum of 40 valid observations were included for analysis. Effect sizes (Cohen's d) were calculated using Borenstein's ${ }^{14}$ equation $d=\ln$ odds ratio $x \frac{\sqrt{3}}{\pi}$. Accelerometery data was included for analysis if a minimum of $30 \mathrm{~min}$ of valid data was recorded during the PE lesson. Outliers were removed for each intensity. Using a 3(intensity)-by-2(complexity) ANOVA, percentage of PE lesson time at moderate (MPA) and vigorous (VPA) intensity was analysed to determine if PE lesson intensity differed significantly between lesson intensity, complexity, and intensity-by-complexity interaction. Cut points for MPA and VPA were taken from a validation study of wrist-worn GENEActiv devices in 120 nine- to ten-year-old children. ${ }^{15}$

This study was funded by the United Kingdom's Economic and Social Research Council (ESRC). The ESRC has not been involved in the design or execution of the study, including data analysis or interpretation.

\section{Results}

Participant flow through the trial is shown in Fig. S2. Percentages of MPA and VPA differed significantly between the six conditions $\left(\mathrm{F}_{\mathrm{MPA}(2)}=5.72, \mathrm{p}<0.01, \mathrm{~F}_{\mathrm{VPA}(2)}=20.91, \mathrm{p}<0.001\right)$, with a main ef-
Table 1

Mean percentage MPA and VPA per PE lesson intensity and complexity.

\begin{tabular}{|c|c|c|c|c|c|}
\hline & & \multicolumn{2}{|c|}{$\begin{array}{l}\% \text { Moderate } \\
\text { PA }\end{array}$} & \multicolumn{2}{|c|}{$\begin{array}{l}\% \text { Vigorous } \\
\text { PA }\end{array}$} \\
\hline & $\mathrm{N}$ & M & $\begin{array}{l}\text { Std } \\
\text { D }\end{array}$ & M & Std D \\
\hline \multicolumn{6}{|l|}{ Intensity } \\
\hline Low & 156 & 9.42 & 4.39 & 2.52 & 2.22 \\
\hline Medium & 148 & 9.91 & 3.18 & 4.53 & 2.59 \\
\hline High & 155 & 11.70 & 4.26 & 7.73 & 3.44 \\
\hline \multicolumn{6}{|l|}{ Complexity } \\
\hline Low & 234 & 7.85 & 2.71 & 4.36 & 3.65 \\
\hline High & 225 & 12.94 & 3.68 & 5.51 & 3.31 \\
\hline \multicolumn{6}{|l|}{ Intensity-by-complexity } \\
\hline Low intensity, low complexity & 77 & 6.71 & 2.21 & 1.50 & 1.70 \\
\hline $\begin{array}{l}\text { Medium intensity, low } \\
\text { complexity }\end{array}$ & 76 & 8.04 & 2.69 & 3.21 & 1.91 \\
\hline High intensity, low complexity & 81 & 8.76 & 2.81 & 8.17 & 2.98 \\
\hline Low intensity, high complexity & 79 & 12.06 & 4.38 & 3.50 & 2.23 \\
\hline $\begin{array}{l}\text { Medium intensity, high } \\
\text { complexity }\end{array}$ & 72 & 11.87 & 2.38 & 5.93 & 2.49 \\
\hline High intensity, high complexity & 74 & 14.91 & 3.10 & 7.24 & 3.85 \\
\hline
\end{tabular}

fects for intensity $\left(\mathrm{F}_{\mathrm{MPA}(2)}=27.86, \mathrm{p}<0.001, \quad \mathrm{~F}_{\mathrm{VPA}(2)}=154.89\right.$, $\mathrm{p}<0.001)$, as well as complexity $\left(\mathrm{F}_{\mathrm{MPA}(1)}=326.63, \mathrm{p}<0.001\right.$, $\left.\mathrm{F}_{\mathrm{VPA}(1)}=26.65, \mathrm{p}<0.001\right)$, indicating that high complexity increased MPA and VPA, and that within each complexity level \%MPA and \%VPA increased as lesson intensity increased (Table 1). Especially MPA was influenced by the increase in complexity, and particularly so for the low intensity lesson, which consisted of juggling. This may be a result of the accelerometers being worn on the wrist.

Table 2 shows the time effect and time-by-condition effects of PE lesson intensity, complexity and intensity-by-complexity. On-task behaviour was significantly higher after $\mathrm{PE}$ than before $\mathrm{PE}$ $\left(F_{(1,51638)}=106.63, \mathrm{p}<0.001, \mathrm{~d}=0.12\right)$. An effect of time-by-intensity on on-task behaviour was found $\left(\mathrm{F}_{(2,51634)}=11.07, \mathrm{p}<0.001\right)$, but only for high intensity PE lessons $\left(\mathrm{t}_{\text {high }}=2.85, \mathrm{p}<0.01, \mathrm{t}_{\text {medium }}=0.52\right.$, $\mathrm{p}=0.60, \mathrm{~d}=0.2$ ) (Fig. 1A). No effect of time-by-complexity on on-task behaviour was observed $\left(\mathrm{F}_{(1,51636)}=1.89, \mathrm{p}=0.17\right)$ (Fig. 1B). The interaction of intensity and complexity was significant $\left(\mathrm{F}_{(2,51628)}=69.19, \mathrm{p}<0.001\right)$.

Table 2

Effect of PE lesson content on on-task behaviour

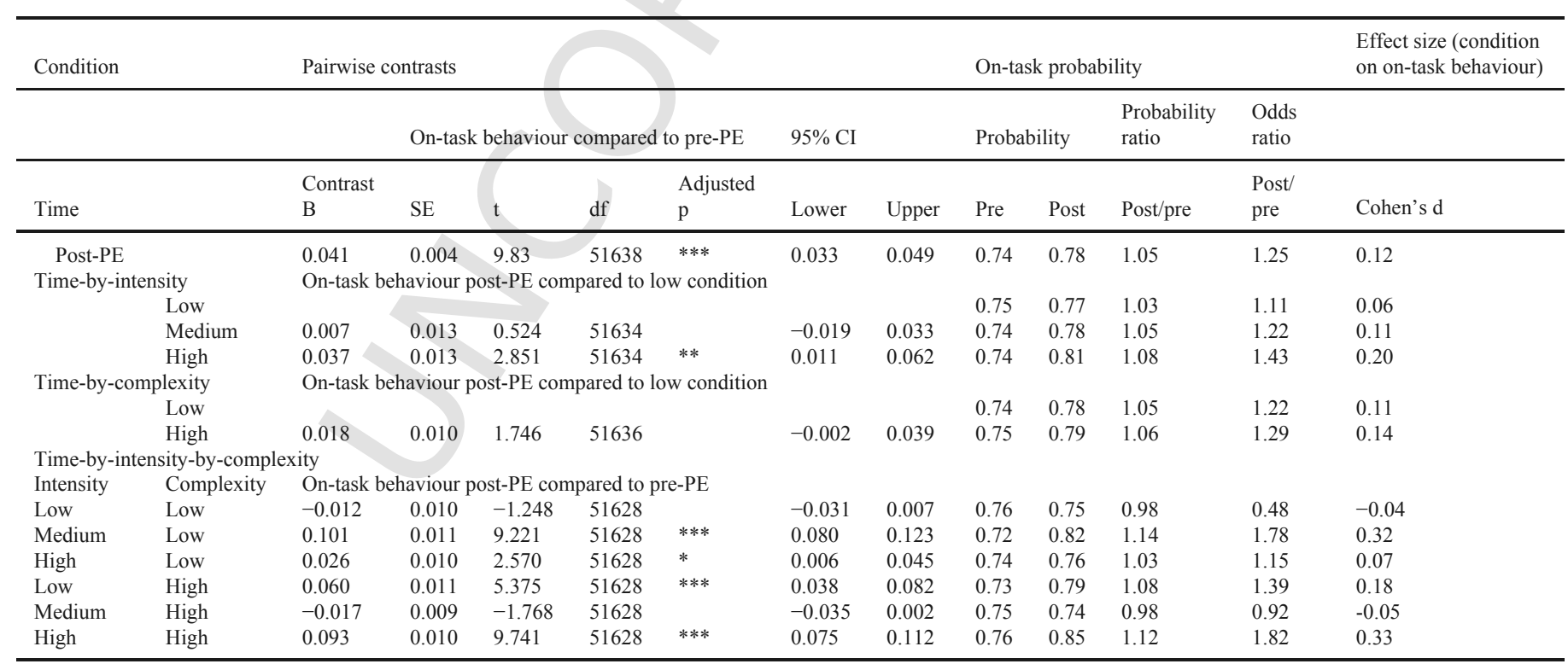




\section{A. Effect size of PE lessons, by intensity}

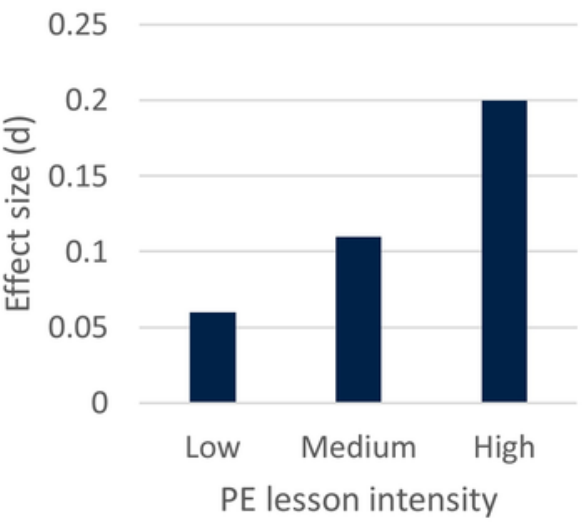

\section{B. Effect size of PE lessons, by complexity}

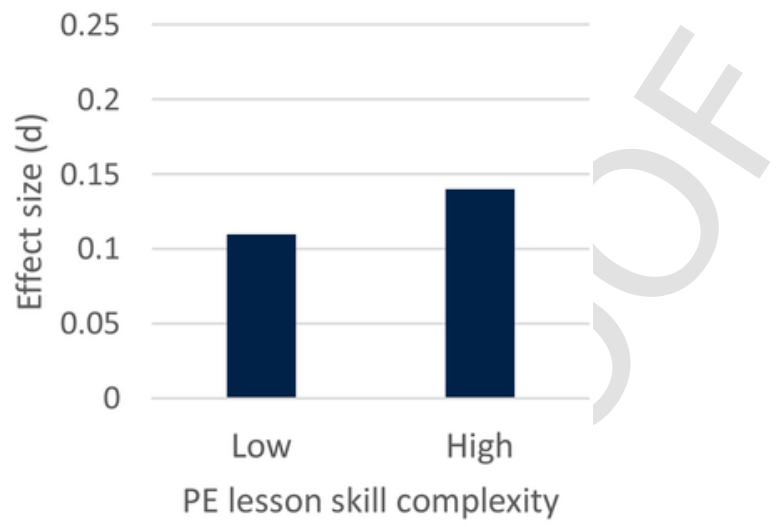

1C. Effect size of PE lessons, by condition

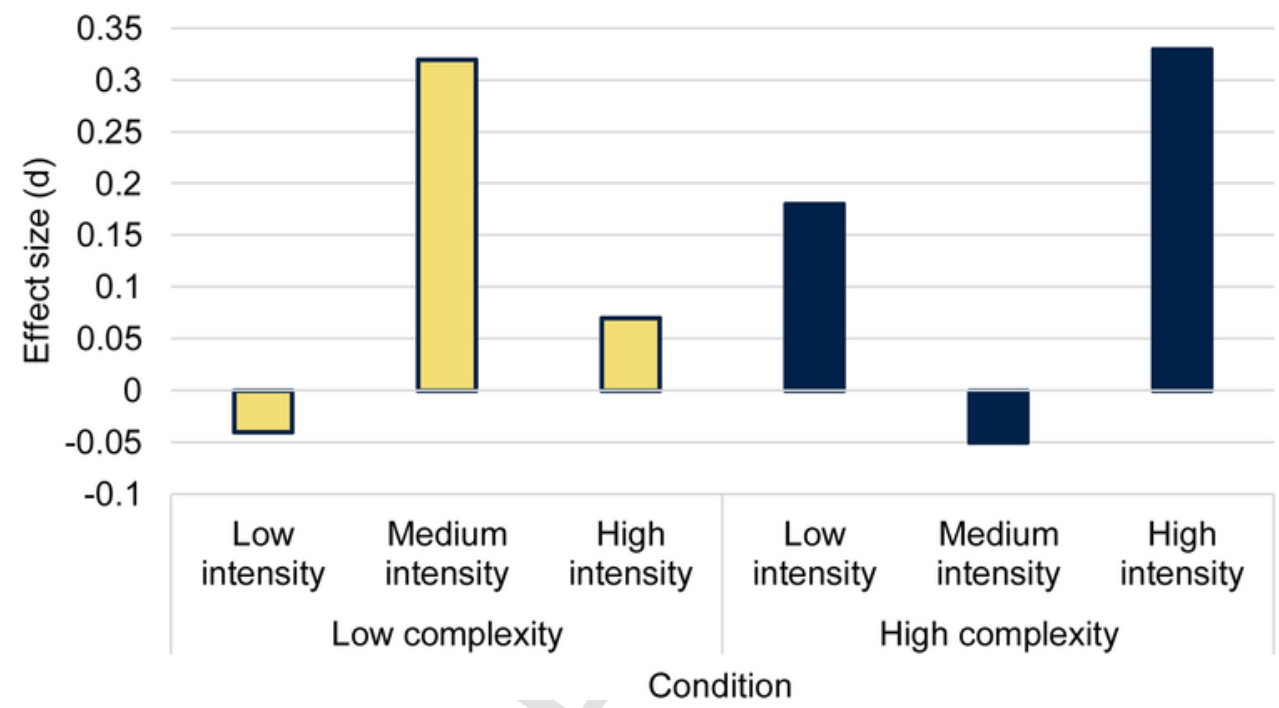

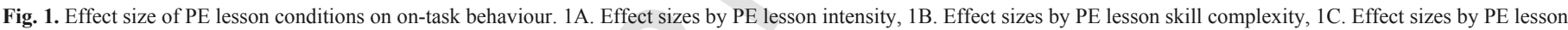
condition.

Fig. 1C shows the effect size for each condition. On-task behaviour did not differ after low intensity/low complexity (control condition) and medium intensity/high complexity PE lessons. After high intensity/low complexity $(\mathrm{OR}=1.12)$ and low intensity/high complexity $(\mathrm{OR}=1.39)$ it differed, but effect sizes were $\mathrm{d}=0.06$ and $\mathrm{d}=0.18 \mathrm{re-}$ spectively. Small positive effect sizes were found for medium intensity/low complexity $(\mathrm{OR}=1.78, \mathrm{~d}=0.32)$ and high intensity/high complexity $(\mathrm{OR}=1.82, \mathrm{~d}=0.33)$.

\section{Discussion}

To our knowledge, this is the first study to systematically compare the effect of six types of PE lessons of varying intensity and complexity on students' on-task behaviour. This study found that after PE lessons, participants displayed equal or increased on-task behaviour compared to before PE, depending on the content of the PE lesson (Fig. 1C).

Our study found an overall effect of PE lesson on on-task behaviour; $d=0.12$, a somewhat smaller effect than Owen et al. ${ }^{16}$ re- ported as the overall association between PA and school engagement in children $(\mathrm{d}=0.27)$. Their review however included studies reporting on wider school engagement rather than specific task-behaviour, and included studies on active classroom lessons and active breaks, as well as PE lessons.

Studies on active classroom breaks or active lessons and subsequent on-task behaviour have reported effect sizes of 0.31 (decreased passive off-task behaviour) ${ }^{17}$ to 0.60 (increased time on-task) ${ }^{18}$ in the same age group as in our study, and active lessons are reported to prevent a decline in on-task behaviour $(\mathrm{d}=-1.28)$ compared to sedentary lessons. ${ }^{19}$ Yet, all of these interventions took place within the classroom. Wilson et al. ${ }^{20}$ investigated active breaks outside the classroom and found no effects on elementary school pupils' subsequent on-task behaviour. Additionally, with active breaks having a shorter duration than PE lessons, and active lessons providing a qualitatively different type of physical activity due to space limitations, these results cannot be directly generalised to PE lessons. PE lesson intervention studies have also reported positive findings on measures of attention. However, to our knowledge no previous studies of PE lessons have 
used on-task behaviour as the outcome measure. Typically, psychometric tests such as the $\mathrm{d} 2$ test of attention, ${ }^{21}$ or Flanker test of inhibition $^{22}$ are used, so these results cannot be directly compared to our study outcomes.

The time-by-intensity effect in our study was significant for high intensity only $(d=0.2)$ (Fig. 1A). This is contrary to our hypothesis that medium intensity PE would have the greatest effect on subsequent behaviour. The difference in our results for intensity may be explained by the use of a different outcome measure to previous studies ${ }^{7}$; our study investigated on-task behaviour during a normal classroom lesson, rather than cognitive performance measured in a laboratory or in a classroom under exam conditions. Additionally, previous studies of exercise intensity have been conducted with adult samples, ${ }^{23,24}$ not pre-adolescents.

Our study found no time-by-complexity effects (Fig. 1B). This is in line with previous studies reporting no effects for cognitively engaging PE lessons on measures of attention, compared to simple PE lessons. ${ }^{22,25}$ This result differs from the positive effects found in studies of on-task behaviour in active lessons. ${ }^{19}$ This may be due to complexity in this intervention being derived from high coordinative demands of the movements in the PE lesson, rather than by adding an academic content to simple movements like in active classroom lessons.

Nonetheless, an effect of time-by-complexity-by-intensity was found. At low complexity, medium intensity had the greatest positive effect on behaviour $(\mathrm{d}=0.32)$, consistent with the hypothesised inverted-U effect of aerobic intensity (Fig. 1C). As studies reporting the inverted-U effect involved exercise on treadmills or stationary bikes, ${ }^{26}$ with little complexity to the movements, it may be that the inclusion of high-complexity exercise in the time-by-intensity analysis influenced the outcome.

As hypothesised, the effects of high-complexity lessons were positive for low and high intensity, with a greater effect for high intensity $(d=0.33)$ than low intensity $(d=0.18)$. Unexpectedly, no effect of medium intensity lessons was found (Fig. 1C). In contrast to previous studies that found positive effects of cognitively challenging moderate intensity exercise compared to simple moderate intensity exercise, ${ }^{10,21,22}$ our study found that at moderate intensity, there was a significant effect of simple, but not cognitively challenging exercise.

As well as the use of on-task behaviour as the outcome measure, the duration of the exercise bout may account for the contrasting result of medium intensity/high complexity PE lessons in our study and previous literature. Finally, there is a possibility that the activities chosen to make the lesson cognitively challenging (passing games using the non-dominant hand, moving and passing, and team games involving passing) affected participants in more ways than just the intensity level and cognitive demands. These were all social activities, requiring work with a partner or team, and in the final activity (games) they competed against their peers. Results from studies reporting games activities are inconclusive; although Pesce et al. ${ }^{27}$ report positive effects of team games on immediate memory performance, Wilson et al. ${ }^{20}$ found no effect of a games intervention on subsequent on-task behaviour. Social interactions and emotional state after PA have been linked to attentional performance, ${ }^{21}$ and may have affected on-task behaviour in our study.

Our study has strong ecological validity due to its settings in normal classroom lessons, and the observation of behaviour in these naturalistic settings. The content of all PE lessons is directly related to the England National Curriculum for PE and required minimal and standard equipment. The PE lessons were delivered by the lead researcher across all participating classrooms to ensure consistency. However, there are some limitations that should be noted. As the same researcher also carried out the observations, and the research assistant observed the PE lessons to take notes of the timings of each activity, there is a potential for bias. PA measures obtained by wrist-worn accelerometers may have been more sensitive to some forms of PA than others (passing versus press-ups) and not give a fully accurate representation of the PE lesson intensity. Finally, the sample size was relatively small. Therefore, a larger-scale cluster randomised controlled trial with blinded assessors is needed to replicate these findings.

\section{Conclusion}

These findings suggest that participation in PE lessons can improve children's on-task behaviour in the classroom. PE lessons involving high complexity and high intensity, or low complexity and medium intensity appear to have the greatest benefits for students' behaviour in the classroom. No detrimental effects of exercise-induced arousal or tiredness on behaviour were found.

\section{Acknowledgements}

This study was funded by the United Kingdom's Economic and Social Research Council (ESRC) [grant number ES/J500112/1]. The ESRC has not been involved in the design or execution of the study, including data analysis or interpretation, and has no influence over the publication of study results.

The authors declare no financial or other interest in the GENEActiv devices used in this study, and have no relations with the manufacturers or distributors.

\section{Appendix A. Supplementary data}

Supplementary material related to this article can be found, in the online version, at doi:https://doi.org/10.1016/j.jsams.2019.12.012.

\section{References}

1. J.E. Donnelly, C.H. Hillman, D. Castelli, et al., Physical activity, fitness, cognitive function, and academic achievement in children: a systematic review, Med Sci Sports Exerc 48 (6) (2016) 1197-1222.

2. C. Álvarez-Bueno, C. Pesce, I. Cavero-Redondo, et al., Academic achievement and physical activity: a meta-analysis, Pediatrics 140 (6) (2017).

3. D. Lubans, J. Richards, C. Hillman, et al., Physical activity for cognitive and mental health in youth: a systematic review of mechanisms, Pediatrics 138 (3) (2016).

4. A. Watson, A. Timperio, H. Brown, et al., Associations between organised sport participation and classroom behaviour outcomes among primary school-aged children, PLoS One 14 (1) (2019), e0209354, [Accessed 2019].

5. C.H. Hillman, K.I. Erickson, A.F. Kramer, Be smart, exercise your heart: exercise effects on brain and cognition, Nat Rev Neurosci 9 (2008) 58-65.

6. J.R. Best, Effects of physical activity on children's executive function: contributions of experimental research on aerobic exercise, Dev Rev 30 (4) (2010) 331-551.

7. Y.K. Chang, J.D. Labban, J.I. Gapin, et al., The effects of acute exercise on cognitive performance: a meta-analysis, Brain Res 1453 (2012) 87-101.

8. J.W. de Greeff, R.J. Bosker, J. Oosterlaan, et al., Effects of physical activity on executive functions, attention and academic performance in preadolescent children: a meta-analysis, J Sci Med Sport 21 (5) (2018) 501-507.

9. Activinsights, GENEActiv instructions, 2012.

10. H. Budde, C. Voelcker-Rehage, S. Pietrabyk-Kendziorra, et al., Acute coordinative exercise improves attentional performance in adolescents, Neurosci Lett 441 (2) (2008) 219-223.

11. Pearson, BOSS user's guide, Pearson, Bloomington, 2013.

12. M.L. McHugh, Interrater reliability: the kappa statistic, Biochem Med 22 (3) (2012) 276-282.

13. IBM SPSS Statistics for windows, version 24.0 [computer program], IBM Corporation, Armonk, NY, 2016.

14. M. Borenstein, L.V. Hedges, J.P.T. Higgins, et al., Converting among effect sizes, in: M. Borenstein (Ed.), Introduction to meta-analysis, John Wiley \& Sons, Chichester, 2009. 
15. S.J. Fairclough, R. Noonan, A.V. Rowlands, et al., Wear compliance and activity in children wearing wrist- and hip-mounted accelerometers, Med Sci Sports Exerc 48 (2) (2016) 245.

16. K.B. Owen, P.D. Parker, B. Van Zanden, et al., Physical activity and school engagement in youth: a systematic review and meta-analysis, Educ Psychol 51 (2) (2016) 129-145.

17. J.K. Ma, L.L. Mare, B.J. Gurd, Classroom-based high-intensity interval activity improves off-task behaviour in primary school students, Appl Physiol Nutr Metab 39 (12) (2014) 1332-1337.

18. M.T. Mahar, S.K. Murphy, D.A. Rowe, et al., Effects of a classroom-based program on physical activity and on-task behavior, Med Sci Sports Exerc 38 (12) (2006) 2086-2094.

19. L.A. Grieco, E.M. Jowers, J.B. Bartholomew, Physically active academic lessons and time on task: the moderating effect of body mass index, Med Sci Sports Exerc 41 (10) (2009) 1921.

20. A. Wilson, T. Olds, K. Lushington, et al., The impact of 10 -minute activity breaks outside the classroom on male students' on-taskbehaviour and sustained attention: a randomised crossover design, Acta Paediatr 105 (4) (2016) E181-E188.

21. M. Schmidt, V. Benzing, M. Kamer, Classroom-based physical activity breaks and children's attention: cognitive engagement works, Front Psychol 7 (2016).

22. M. Schmidt, K. Jager, F. Egger, et al., Cognitively engaging chronic physical activity, but not aerobic exercise, affects executive functions in primary school children: a group-randomized controlled trial, J Sport Exerc Psychol 37 (6) (2015) 575-591.

23. P.D. Tomporowski, Effects of acute bouts of exercise on cognition, Acta Psychol 112 (3) (2003) 297-324.

24. T. McMorris, B.J. Hale, Differential effects of differing intensities of acute exercise on speed and accuracy of cognition: a meta-analytical investigation, Brain Cogn 80 (3) (2012) 338-351.

25. M.C. Gallotta, G.P. Emerenziani, E. Franciosi, et al., Acute physical activity and delayed attention in primary school students, Scand J Med Sci Sports 25 (3) (2015) e331-e338.

26. K. Lambourne, P. Tomporowski, The effect of exercise-induced arousal on cognitive task performance: a meta-regression analysis, Brain Res 1341 (2010) 12-24.

27. C. Pesce, C. Crova, L. Cereatti, et al., Physical activity and mental performance in preadolescents: effects of acute exercise on free-recall memory, Ment Health Phys Act 2 (1) (2009) 16-22. 